\title{
PROPUESTA DE APLICACIÓN DE LOS MAPAS CONCEPTUALES EN UN MODELO PEDAGÓGICO SEMIPRESENCIAL
}

\author{
Alfredo J. Simón Cuevas \\ Instituto Superior Politécnico "José Antonio Echeverría", Cuba
}

\section{INTRODUCCIÓN}

El desarrollo de las tecnologías de la información y las comunicaciones ha favorecido el cambio de los ambientes rutinarios de aprendizaje por otros caracterizados por la innovación y la interacción permanente. El nuevo milenio demanda habilidades o competencias en el manejo de la información por lo tanto los procesos de adquisición, selección y utilización de la misma, así como la creación de nuevos conocimientos, requieren la utilización de herramientas que permitan energizar el proceso de enseñanzaaprendizaje.

En línea con este desafío parece evidente que las universidades, además de la formación regular y de su tradicional actividad de posgrado, deben adoptar otro tipo de medidas que le permitan satisfacer las crecientes necesidades de formación continua. Esta oferta formativa debe garantizar además de gran variedad de programas, flexibilidad de horarios, posibilidad de compatibilizar el estudio con el trabajo, posibilidad de interacción entre profesores y estudiantes haciendo uso de las Tecnologías de la Información y las Comunicaciones (TIC) y de herramientas que potencien el proceso de enseñanza aprendizaje.

Estas organizaciones deben buscar una estructura modular para los contenidos que facilite la flexibilidad en la formación, extender la oferta al mercado internacional y utilizar otras formas complementarias de desarrollo de las materias que no sean las clases tradicionales. Es aquí donde la Educación a Distancia ofrece una solución complementaria y a veces más efectiva para satisfacer las necesidades formativas del mercado objetivo.

Para ofrecer en este entorno un modelo pedagógico que permita de manera eficiente garantizar un aprendizaje efectivo, se ha diseñado en el Centro de Estudios de Ingeniería y Sistemas (CEIS) que pertenece al Instituto Superior Politécnico "José Antonio Echeverría” (CUJAE) un modelo pedagógico que responde a la modalidad de enseñanza Semipresencial, para impartir la Maestría en Informática Aplicada.

En este sistema intervienen 3 actores que son: el estudiante, el tutor y el profesor y está organizado por actividades grupales (en diferentes magnitudes) e individuales, estas pueden ser presenciales 0 a distancia [7].

Para que esto se logre no basta con que el modelo exista y esté correctamente definido, es necesario que los profesores y estudiantes utilicen novedosas técnicas que apoyen el proceso de enseñanza aprendizaje en este esquema en el que es parte esencial que el estudiante logre "aprender a aprender" o "aprenda haciendo" . 
Los Mapas Conceptuales son una técnica que cada día se utiliza más en los diferentes niveles educativos involucrando la asimilación de conceptos y proposiciones nuevas mediante la inclusión en estructuras cognitivas ya existentes, que se conoce como aprendizaje significativo. En los últimos años han alcanzado una gran popularidad y una gran integración con las tecnologías computacionales y de las comunicaciones. Se han convertido en un elemento muy importante en los planes de perfeccionamiento de los sistemas de enseñanza y han extendido su uso a otras esferas de la actividad humana en las que la gestión del conocimiento ocupa un lugar preponderante. Un ejemplo de ello, es el uso en la educación a distancia donde es fundamental el estudio independiente y el autoaprendizaje por parte de los estudiantes.

Con esta investigación el autor se propone demostrar que la inserción de los Mapas Conceptuales en este modelo semipresencial, como forma de representación del conocimiento que de forma incremental adquieren los estudiantes, garantiza la eficiencia en el proceso de enseñanza, en el autoaprendizaje y en la autoevaluación.

\section{LOS MAPAS CONCEPTUALES}

Los Mapas Conceptuales (MC) iniciaron su desarrollo en el Departamento de Educación de la Universidad de Cornell, EUA, durante la década de los setenta y constituyen una respuesta a la teoría del aprendizaje significativo desarrollada por Ausubel, en especial, en lo referente a la evolución de las ideas previas que poseen los estudiantes para lograr un nuevo conocimiento. Los MC han constituido desde entonces, una herramienta de gran utilidad para profesores, investigadores de temas educativos, psicólogos, sociólogos y estudiantes en general, así como para otras áreas sobre todo cuando se necesita tratar con grandes volúmenes de información [5].

Se han publicado diferentes criterios sobre el concepto de los MC, uno de ellos precisamente definido por Novak, su creador, publicado en su texto "Aprendiendo a aprender", define el MC como una técnica que representa, simultáneamente, una estrategia de aprendizaje, un método para captar lo más significativo de un tema y un recurso esquemático para representar un conjunto de significados conceptuales, incluidos en una estructura de proposiciones [6].

Los MC proporcionan un resumen esquemático de lo aprendido, ordenado de diferentes estructuras gráficas. El conocimiento está organizado y representado en todos los niveles de abstracción, situando los más generales e inclusivos en la parte superior, y los más específicos y menos inclusivos en la parte inferior [10].

Los elementos básicos de los Mapas Conceptuales son los siguientes:

Los conceptos: También llamados nodos, hacen referencia a hechos, objetos, cualidades, animales, etc., gramaticalmente los conceptos se pueden identificar como nombres, adjetivos y pronombres.

Las palabras-enlace: Son palabras que unen los conceptos y señalan los tipos de relación existente entre ellos.

Las proposiciones. Están constituidas por conceptos y palabras-enlace. Es la unidad semántica más pequeña que tiene valor de verdad. 
En el MC se organizan dichos elementos, relacionándose gráficamente, y formando cadenas semánticas, es decir cadenas que poseen un significado. Esta manera gráfica de representar los conceptos y sus relaciones provee a los profesores y alumnos de una forma rica para organizar y comunicar lo que saben sobre un tema determinado. Utilizando un sistema de nodos y enlaces, los aprendices dibujan un mapa, que de manera visual representa cómo piensan ellos, donde se relacionan un conjunto de conceptos. Esta representación se modifica con el tiempo a través de la instrucción que reciban o conocimiento que adquieran.

Las características básicas de un Mapa Conceptual [10]:

$\exists \bullet$ Organización del conocimiento en unidades o agrupaciones holísticas, es decir, que cuando se activa una de éstas, también se activa el resto.

$\exists \bullet$ Segmentación de las representaciones holísticas en subunidades interrelacionadas.

- Estructuración serial y jerárquica de las representaciones. En los MC resalta sobre todo la jerarquización.

Tipos de Mapas Conceptuales.

Existen diferentes tipos de MC pero por la propia definición y la razón de ser de estos, los de tipo jerárquico son los más usados y difundidos, además por el acercamiento a la estructura en la que el ser humano almacena el conocimiento.

Los cinco tipos principales son[9]:

1.- Mapas conceptuales en forma de araña: El mapa es estructurado de manera que el término que representa al tema principal es ubicado en el centro del gráfico y el resto de los conceptos llegan mediante la correspondiente flecha.

2.• Mapas conceptuales jerárquicos: la información se representa en orden descendente de importancia. El concepto más importante es situado en la parte superior del mapa.

- Mapa conceptual secuencial: en este tipo de mapa los conceptos son colocados uno detrás del otro en forma lineal.

- Mapa conceptual en sistema: en este tipo de mapa la información se organiza también de forma secuencial pero se le adicionan entradas y salidas que alimentan los diferentes conceptos incluidos en el mapa.

5.- Mapas conceptuales hipermediales: es aquel que en cada nodo de la hipermedia contiene una colección de no más de siete conceptos relacionados entre sí por palabras-enlaces.

\section{LOS MAPAS CONCEPTUALES Y EL APRENDIZAJE}

David Ausubel y Otros [1] formularon una teoría del aprendizaje que ha resultado ser un gran aporte para el perfeccionamiento de la educación. La idea principal en la teoría de Ausubel es que el aprendizaje 
de nuevos conocimientos depende de la medida de lo que ya se conoce. En otras palabras, la construcción del conocimiento comienza con nuestra observación y reconocimiento de eventos y objetos a través de conceptos que ya poseemos.

Otro elemento importante en la teoría de Ausubel, es el enfoque de aprendizaje significativo. Para aprender significativamente los individuos deben relacionar nuevos conocimientos con conceptos relevantes que ellos ya conocen, este tipo de aprendizaje puede ser contrastado con aprendizaje por memorización el que también puede incorporar nueva información a la estructura del conocimiento, pero sin interacción. El aprendizaje significativo es personal, idiosincrásico, e involucra el reconocimiento de relaciones entre conceptos.

El aprendizaje significativo es más eficaz que el memorístico por las razones siguientes[5]:

$\exists \bullet$ porque le afecta en sus tres principales fases: adquisición, retención y recuperación;

$\exists$ - las pruebas realizadas confirman que el enfoque significativo de un material potencialmente significativo hace la adquisición más fácil y más rápida que en el enfoque repetitivo;

$\exists$ • la adquisición significativa es más fácil porque fundamentalmente implica la utilización de estructuras y elementos previamente adquiridos;

- se retiene el conocimiento por un período de tiempo más largo;

La clave del aprendizaje significativo está en relacionar el nuevo material con las ideas ya existentes en la estructura cognitiva del alumno. Por consiguiente, la eficacia de este aprendizaje está en función de su significatividad, no de las técnicas memorísticas (aprendizaje memorístico).

Cañas (profesor del Institute for Human and Machine Cognition of the University of West Florida) y Arguea [2] explican que estudiosos de los procesos internos durante el aprendizaje y las estructuras de memoria han propuesto y sometido a validación modelos que explican cómo aprende el hombre, y cómo almacena lo que aprende, a partir de las teorías del procesamiento de la información.

La memoria es una estructura de conocimientos interrelacionados, la cual esquemáticamente se puede visualizar como una red en la que cada unión (nodo) es un conocimiento y cada flecha la interrelación con otros conocimientos. Aprender, bajo esta perspectiva, se centra en incorporar a la estructura de memoria nuevos aprendizajes y ser capaz de recuperarlos y usarlos cuando se necesita. Enseñar, por consiguiente, se centra en procurar que el aprendiz llene los vacíos existentes en dicha estructura de memoria. Los estudiantes no son receptores pasivos de conocimiento, sino por el contrario, participantes activos en la interpretación de los modelos (muchas veces analogías) que ellos mismos o el profesor les proponen para que intenten aprender aquello que aún no saben.

La concepción constructivista concibe los conocimientos previos del alumno en términos de esquemas de conocimiento. Un esquema de conocimiento se define como "la representación que posee una persona en un momento determinado de su historia sobre una parcela de la realidad'.

Los esquemas de conocimiento incluyen una amplia variedad de tipos de conocimiento sobre la realidad, que van desde informaciones sobre hechos y sucesos, experiencias y anécdotas personales, 
actitudes, normas y valores, hasta conceptos, explicaciones, teorías y procedimientos relativos a dicha realidad. Los esquemas que los alumnos poseen no sólo se caracterizan por la cantidad de conocimientos que contienen, sino también por su nivel de organización interna, es decir, por las relaciones que se establecen entre los conocimientos que se integran en un mismo esquema y por el grado de coherencia entre dichos conocimientos.

Basándose en aprendizaje como procesamiento de información y más específicamente en la línea de Ausubel del aprendizaje significativo, es que Novak introduce el MC como una respuesta al aprendizaje significativo, dentro del marco de un programa denominado "Aprender a Aprender".

Se construye como un proceso[4]:

$\exists \bullet$ Centrado en el alumno y no en el profesor.

$\exists$ - Que atiende al desarrollo de destrezas y no se conforma sólo con la repetición memorística de la información por parte de alumno.

$\exists \bullet$ Que pretenda el desarrollo armónico de todas las dimensiones de la persona, no solamente intelectuales.

Así pues, se trata de una propuesta metodológica de carácter abierto y por tanto, lo importante es la revisión crítica y la adaptación a las necesidades curriculares de cada profesor. Como ya se sabe, no todas las experiencias didácticas tienen los mismos resultados en los distintos grupos y niveles.

Respecto a las destrezas cognitivas, los MC desarrollan [4]:

$\exists \bullet$ Las conexiones con ideas previas, tanto en su confección antes del desarrollo del tema, como en su tratamiento posterior.

$\exists$ • La Capacidad de inclusión, dada la jerarquización de los conceptos y el nivel de comprensión que implica su relación.

$\exists \bullet$ La diferenciación progresiva entre conceptos, sobre todo si se elaboran en diferentes momentos del desarrollo del tema.

$\exists \bullet$ La integración o asimilación de nuevas relaciones cruzadas entre conceptos.

A lo que se quisiera incorporar la capacidad para organizar el conocimiento según una estructuración lógica así como la destreza para formular las proposiciones que definen un segmento de la realidad de una manera concisa y clara. Desde el punto de vista del colectivo contribuye a lograr una mayor uniformidad en el nivel de conocimiento de los integrantes de un grupo.

EI MC aparece como una herramienta de asociación, validación, interrelación, discriminación, descripción y ejemplificación de contenidos, con un alto poder de visualización.

La incidencia de los MC en la pedagogía moderna para definir y organizar planes de estudio, currículo, programas de asignaturas y para la acción directa en el proceso de aprendizaje ha trascendido las aspiraciones iniciales de su creador.

Se puede concluir que los MC tienen una gran importancia en el aprendizaje ya que:

- Facilitan una rápida visualización de los contenidos de aprendizaje. 
- Favorecen el recuerdo y el aprendizaje de manera organizada y jerarquizada.

- Permiten una rápida detección de los conceptos clave de un tema, así como de las relaciones entre los mismos.

- Favorece el desarrollo del pensamiento lógico.

- Los materiales elaborados utilizando MC facilitan el estudio independiente.

- Permiten que el alumno pueda explorar su conocimiento previo acerca de un nuevo tema, así como para la integración de la nueva información que ha aprendido.

- Organiza los conocimientos a partir de las principales relaciones entre los conceptos.

- Favorece el trabajo colaborativo.

\section{APLICACIONES DE LOS MAPAS CONCEPTUALES}

Como se mostró en el epígrafe anterior, la principal aplicación de los mapas conceptuales ha sido en la enseñanza. Ese es su propósito básico, pero las aplicaciones de un MC son amplias. En el caso de las ciencias de la educación, debe destacarse que los MC dirigen la atención, tanto del estudiante como del profesor, sobre el número de ideas importantes en las que deben concentrarse en cualquier tarea específica de aprendizaje.

Los MC resultan muy útiles en las diversas etapas del proceso educativo [3].

- En la planeación, como recurso para organizar y visualizar el plan de trabajo, evidenciar las relaciones entre los contenidos y resumir esquemáticamente el programa de un curso.

- En el desarrollo, como una herramienta que ayuda a los estudiantes a captar el significado de los materiales que pretenden aprender.

- En la evaluación, como recurso para la evaluación formativa. Permite "visualizar el pensamiento del alumno", para así, corregir a tiempo posibles errores en la relación de los conceptos principales.

El MC presenta una serie de características que lo diferencian del esquema tradicional convirtiéndolo en un instrumento mucho más útil, ágil y versátil.

Los aspectos básicos y formales de los MC permiten que el profesor/a pueda utilizarlo para [8]:

$\exists$ - Realizar una presentación inicial del tema o de la unidad, facilitando así que los alumnos incorporen los nuevos conocimientos a un esquema previo.

$\exists \bullet$ Establecer unos límites en los conceptos y relaciones del tema que se deba exponer o desarrollar en clase. El mapa agiliza esta tarea del profesor, al mismo tiempo que facilita el trabajo de comprensión en los alumnos.

- Elaborar una visión global y completa al finalizar el desarrollo de la unidad. 
- Por último, otra de las utilidades más significativas del mapa conceptual para el profesor es la evaluación y seguimiento del aprendizaje del alumno.

El alumno aprende con la utilización del MC a tomar conciencia de sus conocimientos previos, a organizar la nueva información relacionándola con la de temas anteriores, y a elaborar resúmenes y síntesis diferenciando lo fundamental de lo accesorio; todo ello se convierte en una herramienta muy potente para facilitar el recuerdo de todo lo que debe conocer.

EI MC es también un poderoso instrumento para que el alumno aprenda a escribir de forma ordenada, tanto en la elaboración de un discurso como de una frase. Este objetivo se alcanza cuando el alumno realiza la operación inversa a la elaboración del mapa, pasar la información que se representa en un MC a un documento escrito.

También puede ayudar al estudiante a:

$\exists$ - Seleccionar y extraer los elementos más significativos para ubicarlos dentro del mapa.

$\exists \bullet$ Interpretar, comprender e inferir de la lectura realizada.

- Integrar la información en un todo, estableciendo relaciones de subordinación e interrelación.

- Intercambiar puntos de vista sobre una proposición particular, permitiendo ver si es buena, válida, si hacen falta enlaces, y así reconocer la necesidad de un nuevo aprendizaje.

$\exists \bullet$ Organizar el pensamiento y los materiales de estudio.

- Desarrollar ideas y conceptos.

$\exists \bullet$ Expresar el propio conocimiento actual acerca de un tópico.

- Insertar nuevos conceptos en la propia estructura de conocimiento.

Debido a que se alcanza más fácilmente un aprendizaje significativo cuando los nuevos conceptos o significados conceptuales se engloban bajo otros conceptos más amplios, los conceptos más generales e inclusivos se sitúan en la parte superior del mapa, y los conceptos progresivamente más específicos y menos inclusivos, en la inferior. Esta es otra razón por la que los MC más empleados son de tipo jerárquicos.

Teniendo en cuenta que los MC constituyen una representación explícita y manifiesta de los conceptos y proposiciones que posee una persona, ellos facilitan que profesores y alumnos intercambien puntos de vista sobre la validez de un vínculo proposicional determinado, o darse cuenta de las conexiones que faltan entre los conceptos y que sugieren la necesidad de un nuevo aprendizaje. Por esta razón esta técnica ha articulado tan favorablemente con los preceptos de la educación a distancia que supone que estudiantes y profesores no estén físicamente en el mismo lugar y al mismo tiempo. Utilizando tanto la vía sincrónica como la asincrónica, se puede intercambiar desde lejos la interpretación que se tiene sobre un concepto, su definición, alcance y sus relaciones con otros conceptos en un contexto determinado.

Otras aplicaciones son las siguientes: 
En matemáticas los alumnos tienen que enfrentarse a la solución de problemas como "memorización de algoritmos" (sin relacionar conceptos), se enfrentan a conceptos como elementos aislados, o asociados, si se solapan en un problema. Para situaciones como estas, los MC adquieren una importancia relevante, en primer lugar como herramienta metodológica que requiere de forma explícita las relaciones entre los conceptos del alumno, y en segundo lugar como herramienta de control del profesor para detectar las lagunas conceptuales y relacionales de los estudiantes.

Los MC han resultado de gran utilidad en la formulación de problemas de investigación, ya que pueden ser usados para ayudar a personas o grupos en la descripción de sus ideas de forma gráfica. Un proceso estructurado y orientado hacia un aspecto o área de interés. Los MC ayudan a las personas a pensar más efectivamente como grupo sin perder su individualidad.

La riqueza del conocimiento puede ser incrementada por el uso de MC. Diversos autores sugieren que los estudiantes que hacen o analizan MC, tendrán un conocimiento base amplio y, por lo tanto, estarán más preparados para resolver problemas en comparación con aquellos estudiantes que han aprendido por memorización.

Los MC han ido extendiendo su dominio de acción, en un principio aplicados a niveles superiores de enseñanza y pronto adaptaron su elaboración a otros niveles como: primaria y secundaria, incluso en preescolar (algunos autores los denominan mapas preconceptuales) y obedeciendo a diferentes modelos como el semipresencial que propone el autor en esta investigación.

\section{EL MODELO SEMIPRESENCIAL CON EL USO DE LOS MAPAS CONCEPTUALES}

\section{Proceso de impartición de asignaturas}

La impartición de cada una de las asignaturas en este modelo debe hacer especial énfasis en la utilización de los métodos activos de enseñanza-aprendizaje, utilizando así de una manera muy notable el reforzamiento de las interacciones entre los estudiantes. De esta manera, los estudiantes no tienen sólo la responsabilidad de aprender, sino que también tienen la responsabilidad de interactuar con los otros estudiantes para colaborar en el aprendizaje de ellos y servirse de ellos para aprender él mismo. En estas interacciones cada estudiante podrá cumplir entre otros los roles de expositor de un conocimiento dado, evaluador crítico de la exposición de otro estudiante, moderador de la interacción entre dos estudiantes realizando la función de juez. La presentación o exposición, así como la evaluación y discusión dentro del proceso de enseñanza-aprendizaje se realizará tanto en actividades presenciales como en actividades a distancia[7].

\section{Actores en el proceso}

Los actores fundamentales que van a intervenir en el proceso de aprendizaje son[7]:

- Estudiante: un alumno de la asignatura

- Tutor: un graduado de la maestría o un estudiante que ya haya aprobado la asignatura. 
- Profesor: el responsable máximo de la preparación de la asignatura y de su impartición. En cualquier caso, este profesor debe ser miembro del comité de la maestría o estar autorizado por este para poder ejercer esta tarea.

Materiales de trabajo.

Como un elemento básico debe facilitarse a cada estudiante el acceso a materiales impresos o en cualquier otro formato de cada asignatura. Este material debe permitir al estudiante la adquisición de los conceptos básicos que forman el cuerpo teórico de cada asignatura.

Un MC de la asignatura que represente el conocimiento que comprende la misma, donde se muestren los conceptos fundamentales y sus relaciones, sería un material muy didáctico y de gran utilidad para los estudiantes. Este MC sería un resumen de todo el contenido de la asignatura ya que a través de él puede enfatizar lo más significativo de un tema. Además otros materiales importantes para el estudio pudieran ser los diferentes MC que los estudiantes han ido elaborando en el transcurso del curso.

Proceso de aprendizaje [7].

La organización del proceso de aprendizaje está integrada por varias actividades individuales y grupales que pueden ser tanto presenciales como a distancia.

En las primeras actividades presenciales el profesor presentará los contenidos, se crean los grupos de estudio que deben tener entre 3 y 5 integrantes, a los cuales se le asignará un tutor por cada uno de los mismos, para que el trabajo sea más efectivo. Cada alumno estudia una parte del contenido que le corresponde al grupo, luego el grupo se reúne para integrar los resultados del estudio que cada uno hizo y se prepara para la presentación u oponencia de trabajos a otros grupos en las actividades intergrupales. En estas actividades intergrupales los grupos exponen sus trabajos y una oponencia al trabajo realizado por otro equipo. Así se repite un ciclo desde la orientación de otros temas por equipos hasta la presentación las veces que el profesor considere. Al final del ciclo una actividad evaluativa en la que el estudiante demuestre lo aprendido durante el curso.

Como se mencionaba anteriormente y como se ve reflejado en el proceso de aprendizaje, este modelo necesita de métodos más activos de enseñanza-aprendizaje y de herramientas que beneficien la comprensión de los contenidos que se imparten, el auto aprendizaje, el trabajo colaborativo y la evaluación de los conocimientos adquiridos. Una de las herramientas didácticas que engloba todos estos elementos son los MC, sobre todo por los beneficios que reporta al desarrollo del aprendizaje como se explicó en los epígrafes anteriores.

Los MC pueden ser utilizados en cualquiera de los procesos de aprendizaje como por ejemplo:

1. Como recurso para organizar y visualizar el plan de actividades, evidenciar las relaciones entre los contenidos y resumir esquemáticamente el programa del curso.

2. Para representar el conocimiento que se quiere impartir en una actividad, reflejando lo más significativo del tema que se imparte.

3. Para lograr un trabajo más colaborativo entre el estudiante y el profesor, entre el estudiante o grupo de estudiantes y el tutor o entre los grupos. Cuando se construye un MC entre un 
grupo de personas, se está trabajando colaborativamente, todos están contribuyendo a la construcción u organización del conocimiento.

4. Para el uso del profesor, como herramienta para la evaluación del conocimiento adquirido por los estudiantes en la actividad y el seguimiento del aprendizaje de los mismos.

5. En la autoevaluación del estudiante. El estudiante puede hacer una evaluación del conocimiento adquirido construyendo un MC.

Otra aplicación importante que pueden tener los MC en este tipo de modelo es en el diseño curricular del curso. Se pueden asociar las asignaturas creando temas o módulos que agrupen varios nodos de conocimientos, donde cada modulo de estos puede ser un MC y cada concepto del mapa es un nodo de conocimiento. Se entiende como nodo de conocimiento a un grupo de contenidos y habilidades que tienen una estrecha relación entre si (pudiera ser una asignatura). De esta manera se puede hacer una representación de todo el contenido que se pretende impartir. Esta representación donde se encuentran relacionadas todas las asignaturas y a su vez los contenidos que en cada una se trata puede servir para:

1. Determinar cuales son los temas fundamentales que se tratan en el curso

2. Determinar cuales son los puntos en común que pueden existir entre las diferentes asignaturas.

Esta representación en un MC del curso y sobre todo esta última información que manifiesta la misma pudiera mostrar la necesidad o no de hacer algún rediseño del curso para ganar en eficiencia.

\section{CONCLUSIÓN}

Como conclusión de este trabajo podemos decir que:

1. En los modelos semipresenciales es necesario la utilización de herramientas que hagan más activo el aprendizaje y que beneficien el autoaprendizaje y la autoevaluación por parte de los estudiantes.

2. La inserción de los Mapas Conceptuales como herramienta importante para crear, compartir, organizar, almacenar y evaluar el conocimiento, en los diferentes procesos del modelo semipresencial expuesto, contribuirá a que los estudiantes aprendan a aprender, aprendan más y más eficientemente.

\section{RECOMENDACIONES}

El autor recomienda que para la inserción de los MC en este tipo de modelos de enseñanza se elabore alguna metodología o guía de aplicación, que contemple dónde, cómo y cuándo deben ser utilizados. De esta manera su uso se hará de forma más eficiente. 


\section{REFERENCIAS BIBLIOGRÁFICA}

[1] AUSUBEL David., NOVAK Joseph. D., HAINESIAN H. "Psicología Educativa”. México Trillas. 1989.

[2] ARGUEA N., CAÑAS Alberto. J. "Mapas Conceptuales como Herramienta en Estadística Aplicada: Una Propuesta para un Curso a Distancia”, Memoria del IX Congreso Internacional sobre Tecnología y Educación a Distancia, San José, Costa Rica.1998.

[3] DÁVILA Sergio, MARTÍNEZ Guillermo, Curso Taller "Mapas Conceptuales, en busca del aprendizaje significativo", URL: http://umarista.edu.mx/sanluis/lectumc.htm, Universidad Marista, 2000.

[4] DEL CASTILLO José Maria, BARBERÁN Olivares, "Mapas Conceptuales en Matemáticas", URL: http://www.cip.es/netdidactica/articulos/mapas.htm.

[5] FEBles Juan P., ESTRADA Vivian, "Aplicaciones de la Inteligencia Artificial” Primera Edición, Parte III, Mapas Conceptuales, pag. 400, Ed. Universidad de Guadalajara, México, 2002.

[6] PÉREZ DE PRADO Antonio. Tesis de Maestría "Uso de Mapas Conceptuales para la enseñanza de la física", Universidad de Matanzas, UMCC, Cuba, 1999.

[7] ROSETE Alejandro, MACHÍN Omar, "Propuesta de indicaciones metodológicas para la implementación semipresencial de la Maestría en Informática Aplicada del CEIS-CREPIAl”, enero 2003.

[8] SANTILLANA, "El mapa de conceptos y los esquemas en el aula" URL: http://www.indexnet.santillana.es/mapasConceptos/mapasConceptos.htm, 2002.

[9] "Kinds of Concept Maps", URL: http://classes.aces.uiuc.edu/ACES100/Mind/, University of Illinois at Urbana-Champaign, College of Agricultural, Consumer and Environmental Sciences.

[10] "Mapas Conceptuales de Ontoria”, URL: http://venus.javeriana.edu.co/qualitas/, Pontificia Universidad Javeriana, Mayo de 1997. 


\title{
Contactar
}

Revista lberoamericana de Educación

\author{
Principal OEI
}

